\title{
Serotype and genotype distributions of pneumococcal carriage isolates recovered from Brazilian children attending day-care centres
}

\begin{abstract}
Correspondence
Bernard W. Beall

BBeall@cdc.gov
\end{abstract}

Received 14 February 2011

Accepted 30 May 2011
Fabiana C. Pimenta, ${ }^{1,2}$ Maria da Gloria S. Carvalho, ${ }^{1}$ Robert E. Gertz, Jr, ${ }^{1}$ Cristyane G. B. Bastos-Rocha, ${ }^{2}$ Luciana S. C. Oliveira, ${ }^{2}$

Laurine Lacerda Pigosso, ${ }^{2}$ Juliane A. Lima, ${ }^{2}$ Caritas Marquez Franco, ${ }^{3}$ Ana Lucia Andrade ${ }^{2}$ and Bernard W. Beall ${ }^{1}$

\author{
${ }^{1}$ Respiratory Diseases Branch, Centers for Disease Control and Prevention, Atlanta, GA, USA \\ ${ }^{2}$ Institute of Tropical Pathology and Public Health, Federal University of Goiás, Goiânia, Goiás State, \\ Brazil \\ ${ }^{3}$ Secretary of Health, Municipality of Goiânia, Goiás State, Brazil
}

\begin{abstract}
Pneumococcal nasopharyngeal carriage isolates recovered from Brazilian children attending day-care centres in 2005 were assessed for serotype, genotype and penicillin susceptibility phenotype. As 124 of the 253 isolates (49\%) were characterized previously with respect to serotype and penicillin susceptibility, the primary objectives were to examine clonal associations and penicillin susceptibility within major serotypes and to assess the suitability of conventional multiplex PCR for deducing carriage serotypes within this population. Using a combination of PCR-based serotyping and the Quellung reaction, serotypes were identified for $81 \%(205 / 253)$ of the isolates, with serogroups or types 14, 6, 23F, 19F and 18 being predominant. Included within the 205 isolates successfully serotyped by PCR were 28 isolates that had become non-viable. Forty-eight isolates were non-typable using both the PCR method and the Quellung reaction. Penicillin non-susceptibility was observed within 16 of the 18 multilocus sequence types detected. Thus, this study provides further evidence from a diverse collection of pneumococcal clones that PCR-based serotype deduction is useful for providing supportive evidence for pneumococcal conjugate vaccine implementation.
\end{abstract}

\section{INTRODUCTION}

The human nasopharynx (NP) provides a reservoir for pneumococcal strains that cause disease (de Lencastre \& Tomaz, 2002), and elucidating capsular serotype distribution within carriage is useful for monitoring vaccine suitability and vaccination impact. In addition, the determination of penicillin-susceptibility profiles among NP isolates potentially provides some assessment of recent $\beta$-lactam antibiotic usage within a given region as these drugs directly select for the emergence of detectable penicillin non-susceptibility in pneumococcal populations (Dowson et al., 1994).

Pneumococci are traditionally serotyped by the Quellung reaction; however, sequential multiplexed PCR approaches based on serotype-specific DNA sequences provide a viable and straightforward alternative (Pai et al., 2006; Carvalho et al., 2010). Multilocus sequence typing (MLST) and

Abbreviations: MLST, multilocus sequence typing; NP, nasopharynx; NT, non-typable; ST, sequence type. penicillin-susceptibility testing allow the global tracking of specific pneumococcal clones as causes of disease or carriage and the detection of emerging resistance to $\beta$-lactam antibiotics. Here, we describe the distribution of serotypes and penicillin-resistance phenotypes and genotypes among pneumococcal NP carriage isolates previously recovered from healthy Brazilian children, and show that multiplex PCR provides a useful alternative for revealing serotype distribution among these diverse strains.

\section{METHODS}

A collection of 217 viable Streptococcus pneumoniae nasopharyngeal swab specimen isolates were recovered from 686 stored isolates originally collected from 1192 healthy children $\leqslant 5$ years old attending 62 day-care centres in the municipality of Goiânia, Brazil, in 2005 (Franco et al., 2010). The remaining 469 of these isolates were not viable after storage. From the latter, we randomly selected 36 stored cultures for PCR-based serotype determination. Of the 253 isolates (217 viable and 36 non-viable) examined in our study at the Centers for Disease Control and Prevention (CDC), serotype and 
resistance properties of 124 (49\%) have been described previously (Franco et al., 2010; data compared in Results).

All testing described here was performed in the CDC Streptococcus Laboratory. Pneumococci were identified by colony morphology, Gram staining, optochin test and bile solubility test (Franco et al., 2010). Susceptibility for penicillin was assessed using an oxacillin disc test $(\geqslant 19 \mathrm{~mm})$ and MIC methods. The MIC breakpoints for penicillin were $\leqslant 0.06 \mu \mathrm{g} \mathrm{ml}^{-1}$ (susceptible), $0.12-1.0 \mu \mathrm{g} \mathrm{ml} \mathrm{m}^{-1}$ (intermediate resistance) and $\geqslant 2.0 \mu \mathrm{g} \mathrm{ml}^{-1}$ (resistant) (CLSI, 2007).

For the 217 viable isolates, crude DNA extraction and sequential multiplex PCR for 29 serotypes commonly associated with pneumococcal disease were performed as described previously (Pai et al., 2006). For the 36 non-viable isolates available for analyses, $500 \mu \mathrm{l}$ of the resuspended stored specimen was centrifuged and also subjected to crude DNA extraction. The multiplex reactions used for this work (29 individual reactions) were redistributed to target common invasive and carriage serotypes observed in Latin America (Laval et al., 2006; Dias et al., 2007). All multiplex reactions included corresponding serotype controls and the internal pneumococcal identification control cpsA. For viable isolates, the Quellung reaction, employing CDC typing sera, was used for final serotype resolution from isolates PCR typed as $6 \mathrm{~A} / 6 \mathrm{~B} / 6 \mathrm{C} / 6 \mathrm{D}, 22 \mathrm{~A} / 22 \mathrm{~F}$ and serogroup 18. A $w c i N 6^{\mathrm{C}}$-specific PCR was performed to identify potential serotype
6C and 6D isolates, as described previously (Carvalho et al., 2009; Bratcher et al., 2010). Classical serotyping, including the Quellung reaction, for assigning the specific serotype was used to assess viable isolates that were non-serotypable by PCR.

MLST was performed as described on the global pneumococcal MLST database (http://spneumoniae.mlst.net) with modifications (Moore et al., 2008) on 55 viable isolates, representing 12 detected serotypes and a random selection of non-typable (NT) isolates. These isolates were all reconfirmed for penicillin-susceptibility phenotypes using the broth dilution method (CLSI, 2007).

\section{RESULTS AND DISCUSSION}

Capsular serotypes or serogroups were assigned for 177/217 $(81.6 \%)$ of the viable isolates and for $28 / 36(77.8 \%)$ of the non-viable stored isolates (Table 1). Forty-eight of the 253 isolates $(19.0 \%)$ were NT using PCR, including 40 viable isolates that were also NT using the classical Quellung methodology for all known serotypes. The cpsA positive control was detected in only $12(25 \%)$ of the 48 NT isolates. Serotype distribution was similar to that in the

Table 1. Serotype, penicillin MICs and genotype (ST) distribution of pneumococci isolated from the NP of healthy children $\leqslant 5$ years old attending day-care centres in Brazil

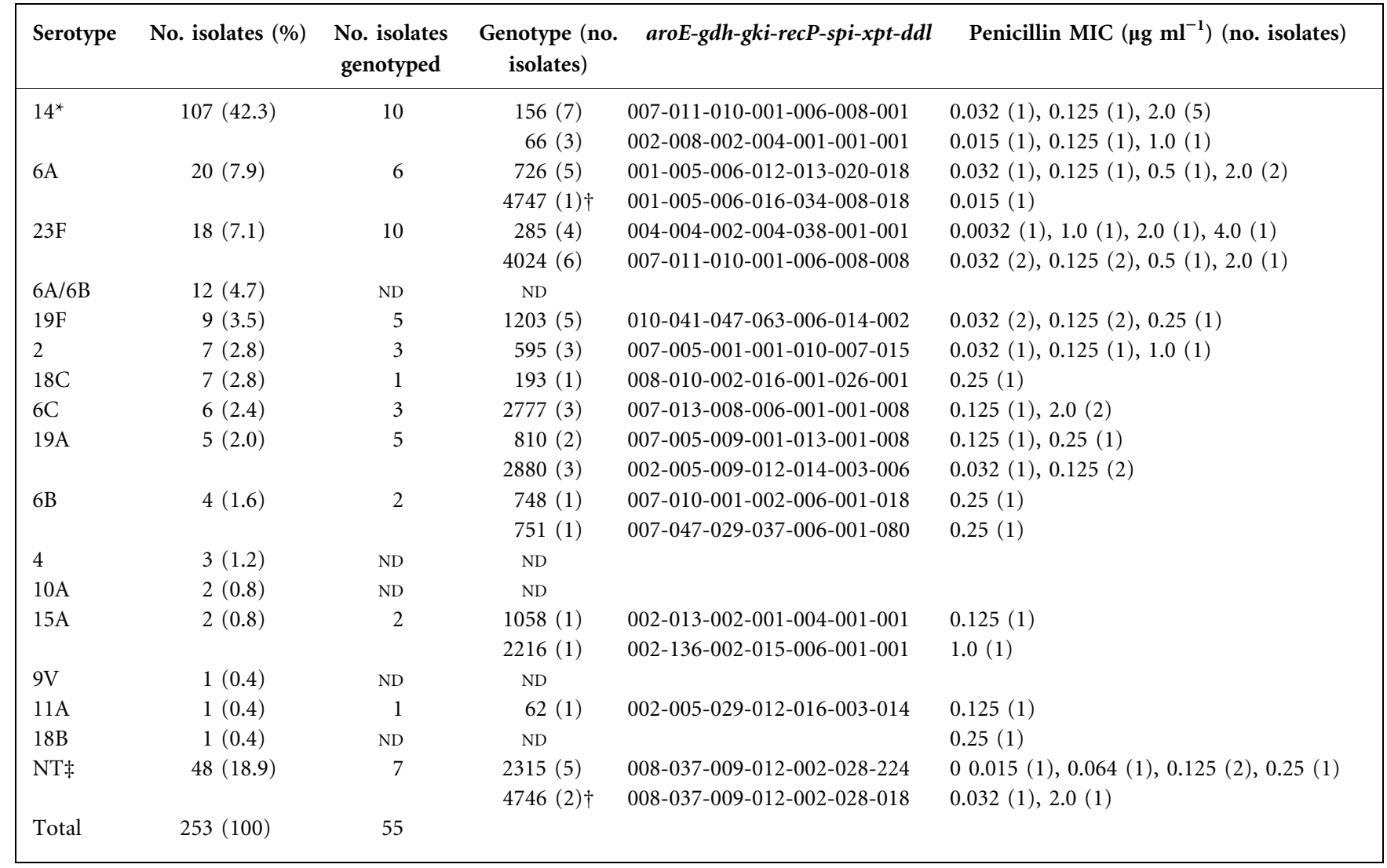

ND, Not done (non-viable stored isolates).

${ }^{\star}$ Three isolates tested as serotype 14 by Quellung reaction consistently yielded the mixed multiplex PCR result of serotype 14 and serotype $6 \mathrm{~A} / 6 \mathrm{~B}$, despite repeated isolation attempts to resolve this.

$\dagger$ New STs discovered during this work.

$\$ N T$, Non-typable by conventional multiplex PCR and Quellung reaction. 
recently published overlapping study (Franco et al., 2010) in that serotypes/serogroups 14, 23F, 19A, 6 and 19F were the most common serotypes. However, we did find discrepancies among nine $(7.3 \%)$ of the 124 results that were tested in both studies. Three isolates that were found previously to be NT were serotypes 14 (two isolates) and 6B (one isolate). The other six discrepancies comprised serotypes 19A (previously 19F), 19F (previously 19A), 14 (previously 23F), 6C (previously 6B) and two NT isolates (previously $6 \mathrm{~A}$ and $6 \mathrm{~B}$ ). Although the previously published study (Franco et al., 2010) reported the detection of 46 different serotypes, among our smaller sampling we detected only 16 (in addition to NT isolates). It is possible that our study was biased due to the fact that we primarily characterized viable isolates, which were in the minority among the collection. It is also possible that there were differences in serotyping methodologies. As described in Methods, 217 of the 253 isolates that we characterized were from an overall collection of 686 stored isolates, 469 $(68.4 \%)$ of which did not maintain viability during storage. It is conceivable that much of the original serotype diversity reflected in the original study was lost, as we have noted profound differences in the abilities of different pneumococcal strains to maintain viability under different environmental conditions.

Using the first multiplex reaction for serotypes $6 \mathrm{~A} / 6 \mathrm{~B} / 6 \mathrm{C} /$ (6D), $9 \mathrm{~V} /(9 \mathrm{~A}), 14,19 \mathrm{~A}$ and $23 \mathrm{~F}$ (note that serotypes rarely encountered in carriage or disease are in parentheses), serotypes or serogroups were deduced for $171(67.6 \%)$ of the isolates; and using the second reaction for serotypes 3 , $7 \mathrm{~F} /(7 \mathrm{~A}), 10 \mathrm{~A}, 15 \mathrm{~B} / 15 \mathrm{C}, 18 \mathrm{C} /(18 \mathrm{~A}) /(18 \mathrm{~B}) /(18 \mathrm{~F})$ and $19 \mathrm{~F}$, serotypes or serogroups were deduced for an additional 19 $(7.5 \%)$ isolates. Sequential PCRs 3-6 [reaction 3 comprised $1,5,9 \mathrm{~N} /(9 \mathrm{~L}), 11 \mathrm{~A} /(11 \mathrm{D})$ and $17 \mathrm{~F}$; reaction 4 comprised 4 , $7 \mathrm{C} /(7 \mathrm{~B}) /(40), 12 \mathrm{~F} /(12 \mathrm{~A}) /(44) /(46), 23 \mathrm{~A}$ and $38 /(25 \mathrm{~A}) /(25 \mathrm{~F})$; reaction 5 comprised $2,8,20,22 \mathrm{~F} /(22 \mathrm{~A}), 31$ and 34 ; reaction 6 comprised $15 \mathrm{~A} /(15 \mathrm{~F}), 16 \mathrm{~F}, 33 \mathrm{~F} /(33 \mathrm{~A}) / 37,35 \mathrm{~B}$ and $35 \mathrm{~F} /(47 \mathrm{~F})$ ] added an additional 15 isolates that were PCR serotyped, giving a total of $205(81.0 \%)$ of the 253 isolates. The cpsA pneumococcal identification control was not detected in four serotype 14 isolates. Using the Quellung reaction, we verified that these strains expressed serotype 14. The most frequent types or serogroups recovered were serotype 14 , serogroup 6 , serotype $23 \mathrm{~F}$, serotype $19 \mathrm{~F}$ and serogroup 18 , accounting for 183 $(72.3 \%)$ of the isolates. The absence of the $c p s A$ amplicon positive control was much more common among NT isolates, occurring in $75 \%(36 / 48)$ of the NT isolates. Remarkably, none of the 40 viable Quellung NT isolates corresponded to a 'PCR serotype'. We have found that most invasive NT isolates yield a PCR-derived serotype, indicating that non-typeability among invasive isolates could possibly reflect loss of capsule expression after recovery from invasive infections.

Mixed carriage of two to three serotypes was detected in 17 children, involving the combinations $14+6 \mathrm{~A} / 6 \mathrm{~B}+15 \mathrm{~A}$ (two children), $14+6 \mathrm{~A} / 6 \mathrm{~B}+19 \mathrm{~A}$ (one), $14+6 \mathrm{~A} / 6 \mathrm{~B}+18 \mathrm{C}$ (one), $14+6 \mathrm{~A} / 6 \mathrm{~B}+23 \mathrm{~F}$ (one), $14+6 \mathrm{~A}$ (six), $14+6 \mathrm{~B}$ (one), $14+6 \mathrm{C}$ (one), $14+18 \mathrm{C}$ (one), $14+9 \mathrm{~L} / 9 \mathrm{~N}$ (one), $14+6 \mathrm{~A} / 6 \mathrm{~B}$ (one) and $19 \mathrm{~F}+6 \mathrm{~A} / 6 \mathrm{~B}$ (one). Three of the six $6 \mathrm{~A} / 6 \mathrm{~B}$ isolates were $w c i N 6^{\mathrm{CD}}$ negative but were non-viable, so could not be resolved into component serotypes $6 \mathrm{~A}$ and 6B using our PCR assay. Curiously, three viable isolates were found to be PCR positive for 6A/6B and PCR negative for $w c i N 6^{\mathrm{CD}}$, yet were also PCR positive for serotype 14 . These three isolates were Quellung positive for serotype 14, yet Quellung negative for serogroup 6, despite repeated restreaking and testing of multiple single colonies by both the Quellung reaction and PCR. Presumably, these three isolates reflect the expression of a functional cps14 locus and the presence of non-functional serogroup 6-specific capsular biosynthetic locus sequences. We have not encountered this result previously among thousands of diverse isolates that have been serotyped in parallel using both the Quellung reaction and PCR (unpublished data). A mix of serotypable and non-serotypable pneumococci were found in three children: 14+NT (two isolates) and 6A/ $6 \mathrm{~B}+\mathrm{NT}$ (one non-viable isolate). It was interesting that serotype 14 and serogroup 6 were over-represented among the 20 mixed-culture specimens (18 and 15, respectively).

Our strategy was to perform MLST within each serotype encountered that exhibited any penicillin non-susceptibility whenever possible. We targeted representative penicillinsensitive (MIC 0.015-0.064 $\mu \mathrm{g} \mathrm{ml}^{-1}$ ), penicillin-intermediate (MIC $0.125-1.0 \mu \mathrm{g} \mathrm{ml}^{-1}$ ) and penicillin-resistant (MIC $\geqslant 2 \mu \mathrm{g} \mathrm{ml}^{-1}$ ) isolates within each serotype whenever possible. Thirteen $(23.6 \%)$ of 55 genotyped isolates were penicillin resistant (MIC $\geqslant 2 \mu \mathrm{g} \mathrm{ml}^{-1}$ ) and $28(50.9 \%)$ isolates had intermediate penicillin resistance (MIC 0.125$1.0 \mu \mathrm{g} \mathrm{ml}^{-1}$ ). Among these, we found 18 multilocus sequence types (STs) (Table 1), two of which had not been identified previously. In our experience, we have found that pneumococcal strains of the same ST and serotype generally share a narrow range of penicillin MIC values (Gertz et al., 2003). It was therefore interesting in this study to reproducibly observe a broad range of penicillin MICs (exhibiting up to 66-fold differences) within 11 of the $12 \mathrm{ST} /$ serotype combinations represented by multiple isolates (Table 1 ). The observation of intermediate penicillin resistance within STs 62, 66 and 193 is possibly reflective of high antibiotic use within this community, as these STs are normally associated with basally penicillin-sensitive strains (Gertz et al., 2003; Beall et al., 2006). Several of these STs or ST/serotype combinations were interesting in that they have been documented exclusively from Brazil and other Latin American countries within the global pneumococcal MLST database (http://spneumoniae. mlst.net; unless specific references are provided regarding MLST data presented here, the source of the information provided is the global database). These included STs 726, 748, $810,1751,2777,2880,4746$ and 4747 (this work or previously recorded work). ST/serotype associations unique to Brazil or other South American countries include ST595/serotype 2, ST2777/6C, ST193/18C, ST1058/15A, ST2216/15A, ST810/ 19A, ST285/22F and ST4024/23F. New STs encountered 
during this work included ST4747 and ST4746. Many of the remaining ST/genotype associations depicted in Table 1 also differed from that typically encountered in the USA and elsewhere. For example, among the ten serotype 14 isolates that were genotyped, we found that ST156 was predominant. ST156 is associated primarily with globally disseminated penicillin-resistant serotype 9V (McGee et al., 2001) and is also the most common invasive serotype $9 \mathrm{~V}$ genotype in the USA (Beall et al., 2006). The remaining serotype 14 isolates were ST66, usually associated with penicillin-susceptible serotype 9N in the USA (Gertz et al., 2003; Beall et al., 2006) and elsewhere. The clonal complex represented by ST156 was also well represented by serotype 23F. Six of the nine serotype $23 \mathrm{~F}$ isolates were ST4024, a single-locus variant of ST156. It is interesting that ST4024 was initially discovered within a single invasive serotype 19A isolate recovered in the USA from a child in 2006 (Beall et al., 2011).

Eleven serogroup 6 isolates were genotyped, with no interserotype genetic relatedness detected between the three detected serotypes $(6 \mathrm{~A}, 6 \mathrm{~B}, 6 \mathrm{C})$. We have recently shown that we are capable of resolving all four known serogroup 6 serotypes, including 6D (Mercado et al., 2011), using our specific factor sera prepared against serotypes $6 \mathrm{~A}, 6 \mathrm{~B}$ and $6 \mathrm{C}$ (Melnick et al., 2010).

For the serotypes found in this study, only serotype 2 was not included among the 29 individual PCR assays that constituted the sequential multiplex assay used (Pai et al., 2006). We have since expanded the number of individual reactions to 40 (see http://www.cdc.gov/ncidod/biotech/ files/pcr-Latin-Amer-clinical-specimens.pdf for the Latin American sequential multiplex assay), such that the current assay includes serotype 2 and ten additional serotypes that we have found to be well represented in other carriage studies. There were a large number of isolates (48) that were NT using the Quellung reaction and PCR methods. It appears that the majority of these NT isolates are likely to represent genotypes within the large ST344 lineage, as both STs that were detected (ST2315 in five isolates and ST4746 in two isolates) were found to be triple-locus variants of ST344. ST344 is the ancestral strain of a large pneumococcal lineage that has lost its capsular locus, is commonly associated with carriage and is the most commonly implicated pneumococcal genotype in conjunctivitis outbreaks (Hanage et al., 2006).

For serotype 14 and NT isolates, our genotyping results were in general agreement with those described recently for yet another overlapping study that analysed some of the same isolates (Andrade et al., 2010). These authors performed PFGE analysis on 106 carriage isolates comprising 84 serotype 14 isolates and $22 \mathrm{NT}$ isolates. They found that the major cluster of serotype 14 isolates shared similar PFGE patterns to ST156 strains. In addition, they found that the majority of their NT strains shared the same multilocus ST, ST2315, which we also found among five of the seven NT isolates from our study. They did not, however, point out the relatedness of ST2315 within the major NT lineage with the founder genotype ST344. This is also evident when performing eBURST on the entire known database of 6500 STs (as of 11 May 2011) allowing for ST sets to share five of seven identical alleles for inclusion within the same eBURST group. When doing so, both ST2315 and ST4716 (found among two of our NT isolates) were within the same 82 -member group predicted to have originated from the NT ST344 clone (see http:// spneumoniae.mlst.net/eburst/database.asp).

Although multiple strains of pneumococci often colonize the NP simultaneously, the identification of multiple serotypes from a single specimen is often unsuccessful. The study presented here was limited in that it did not utilize more sensitive methodology recently developed that uses broth enrichment of NP swabs (Carvalho et al., 2010). Broth enrichment was found to improve pneumococcal isolation on solid medium and was especially useful for detection of multiple serotypes within mixed broth cultures without pneumococcal isolation. When this methodology is utilized, it is essential to carry out all of the multiplex reactions, even after encountering positives within earlier reactions. It is extremely difficult to detect mixed serotypes using conventional isolation-based methodology due to the predominant serotype masking the presence of less densely colonizing serotypes. Nonetheless, using conventional Quellung-based serotyping and multiplex PCR on isolates, we succeeded in identifying mixed carriage from $20 \mathrm{NP}$ swabs. PCR-based serotype assignment of 36 non-viable cultures allowed us to assign serotypes to 28 additional isolates, with 8 isolates found to be PCR-nontypable (cpsApositive but lacking serotype-specific amplicons).

In this study, we targeted the most prevalent pneumococcal serotypes reported to be found in Brazil with the PCR serotyping assay. Through the use of the first reaction, we could serotype nearly $70 \%$ of the isolates, which greatly streamlined our efforts and reduced costs. The serotype distribution among these carriage isolates in our study was similar to that found during previous invasive (Dias et al., 2007) and carriage (Laval et al., 2006; Franco et al., 2010) studies in Brazil, which collectively predict excellent coverage by the conjugate vaccines PCV7, PCV10 and PCV13. Pneumococcal serotypes found during this study that are covered by PCV7 and PCV10 (14, 6A or $6 \mathrm{~B}, 23 \mathrm{~F}$, 19F, 18C, 4 and 9V) represented 181 (71.5\%) isolates. When including the additional serotypes (6C and 19A) predicted to be covered by PCV13 (Dinleyici, 2010), this percentage increases to $75.9 \%$ (192 isolates). Among PCV7-related types, serotypes 6A, 6C and 19A were prevalent. Recent studies have documented an increase in the incidence of serotype 19A invasive disease (Moore et al., 2008) and, to a relatively small degree, an increase in 6C invasive disease (Park et al., 2008). Our results further support the contention that multiplex PCR is a reliable method for serotyping genetically diverse pneumococcal carriage isolates (or stored non-viable pneumococcal cultures) and should prove useful for evaluating serotype 
distribution in support of pneumococcal conjugate vaccine introduction in Brazil.

\section{ACKNOWLEDGEMENTS}

The authors thank Zhongya Li, Alexis Roundtree and Yusra Ahmad (CDC Streptococcus Laboratory) for their help with MLST. This study was supported in part by the Brazilian National Research Council (CNPq-301646/2006-6 and 473880/2006-7) and American Society for Microbiology (Latin American Fellowship, 2007). A. L. A. is a research fellow of the Brazilian Council for Scientific and Technological Development (CNPq) (grant no. 306096/2010-2).

\section{REFERENCES}

Andrade, A. L., Franco, C. M., Lamaro-Cardoso, J., André, M. C., Oliveira, L. L., Kipnis, A., Rocha, C. G., Andrade, J. G., Alves, S. L. \& Park, I. H. (2010). Non-typeable Streptococcus pneumoniae carriage isolates genetically similar to invasive and carriage isolates expressing capsular type 14 in Brazilian infants. J Infect 61, 314-322.

Beall, B., McEllistrem, M. C., Gertz, R. E., Jr, Wedel, S., Boxrud, D. J., Gonzalez, A. L., Medina, M. J., Pai, R., Thompson, T. A. \& other authors (2006). Pre- and postvaccination clonal compositions of invasive pneumococcal serotypes for isolates collected in the United States in 1999, 2001, and 2002. J Clin Microbiol 44, 999-1017.

Beall, B. W., Gertz, R. E., Jr, Hulkower, R. L., Whitney, C. G., Moore, M. R. \& Brueggemann, A. B. (2011). Shifting genetic structure of invasive serotype 19A pneumococci in the United States. J Infect Dis 203, 1360-1368.

Bratcher, P. E., Kim, K. H., Kang, J. H., Hong, J. Y. \& Nahm, M. H. (2010). Identification of natural pneumococcal isolates expressing serotype $6 \mathrm{D}$ by genetic, biochemical and serological characterization. Microbiology 156, 555-560.

Carvalho, M. G., Pimenta, F. C., Gertz, R. E., Jr, Joshi, H. H., Trujillo, A. A., Keys, L. E., Findley, J., Moura, I. S., Park, I. H. \& other authors (2009). PCR-based quantitation and clonal diversity of the current prevalent invasive serogroup 6 pneumococcal serotype, 6C, in the United States in 1999 and 2006 to 2007. J Clin Microbiol 47, 554-559.

Carvalho, M. G., Pimenta, F. C., Jackson, D., Roundtree, A., Ahmad, Y., Millar, E. V., O'Brien, K. L., Whitney, C. G., Cohen, A. L. \& Beall, B. W. (2010). Revisiting pneumococcal carriage by use of broth enrichment and PCR techniques for enhanced detection of carriage and serotypes. J Clin Microbiol 48, 1611-1618.

CLSI (2007). Performance Standards for Antimicrobial Susceptibility Testing; Approved Standard, 16th Informational Supplement. M100S17. Wayne, PA: Clinical and Laboratory Standards Institute.

de Lencastre, H. A. \& Tomaz, A. (2002). From ecological reservoir to disease: the nasopharynx, daycare centers and drug-resistant clones of Streptococcus pneumoniae. J Antimicrob Chemother $\mathbf{5 0}$ (Suppl. S3), 7582.
Dias, C. A., Teixeira, L. M., Carvalho, M. G. \& Beall, B. (2007). Sequential multiplex PCR for determining capsular serotypes of pneumococci recovered from Brazilian children. J Med Microbiol 56, 1185-1188.

Dinleyici, E. C. (2010). Current status of pneumococcal vaccines: lessons to be learned and new insights. Expert Rev Vaccines 9, 10171022.

Dowson, C. G., Coffey, T. J. \& Spratt, B. G. (1994). Origin and molecular epidemiology of penicillin-binding-protein-mediated resistance to $\beta$-lactam antibiotics. Trends Microbiol 2, 361-366.

Franco, C. M., Andrade, A. L. S. S., Andrade, J. G., Almeida e Silva, S., Oliveira, C. R., Pimenta, F. C., Lamaro-Cardoso, J., Brandão, A. P., Almeida, S. C. \& other authors (2010). Survey of nonsusceptible nasopharyngeal Streptococcus pneumoniae isolates in children attending day-care centers in Brazil. Pediatr Infect Dis J 29, 77-79.

Gertz, R. E., Jr, McEllistrem, M. C., Boxrud, D. J., Li, Z., Sakota, V., Thompson, T. A., Facklam, R. R., Besser, J. M., Harrison, L. H. \& other authors (2003). Clonal distribution of invasive pneumococcal isolates from children and selected adults in the United States prior to 7valent conjugate vaccine introduction. J Clin Microbiol 41, 4194-4216.

Hanage, W. P., Kaijalainen, T., Saukkoriipi, A., Rickcord, J. L. \& Spratt, B. G. (2006). A successful, diverse disease-associated lineage of nontypeable pneumococci that has lost the capsular biosynthesis locus. J Clin Microbiol 44, 743-749.

Laval, C. B., de Andrade, A. L., Pimenta, F. C., de Andrade, J. G., de Oliveira, R. M., Silva, S. A., de Lima, E. C., Fabio, J. L., Casagrande, S. T. \& Brandileone, M. C. (2006). Serotypes of carriage and invasive isolates of Streptococcus pneumoniae in Brazilian children in the era of pneumococcal vaccines. Clin Microbiol Infect 12, 50-55.

McGee, L., McDougal, L., Zhou, J., Spratt, B. G., Tenover, F. C., George, R., Hakenbeck, R., Hryniewicz, W., Lefévre, J. C. \& other authors (2001). Nomenclature of major antimicrobial-resistant clones of Streptococcus pneumoniae defined by the pneumococcal molecular epidemiology network. J Clin Microbiol 39, 2565-2571.

Melnick, N., Thompson, T. A. \& Beall, B. W. (2010). Serotype-specific typing antisera for pneumococcal serogroup 6 serotypes $6 \mathrm{~A}, 6 \mathrm{~B}$, and 6C. J Clin Microbiol 48, 2311-2312.

Mercado, E., Srinivasan, V., Hawkins, P., Chochua, S., Ochoa, T., Beall, B. \& McGee, L. (2011). First report of Streptococcus pneumoniae serotype 6D in South America. J Clin Microbiol 49, 2080-2081.

Moore, M. R., Gertz, R. E., Jr, Woodbury, R. L. G., Barkocy-Gallagher, G. A., Schaffner, W., Lexau, C., Gershman, K., Reingold, A., Farley, M. \& other authors (2008). Population snapshot of emergent Streptococcus pneumoniae serotype 19A in the United States, 2005. $J$ Infect Dis 197, 1016-1027.

Pai, R., Gertz, R. E., Jr \& Beall, B. (2006). Sequential multiplex PCR approach for determining capsular serotypes of Streptococcus pneumoniae isolates. J Clin Microbiol 44, 124-131.

Park, I. H., Moore, M. R., Treanor, J. J., Pelton, S. I., Pilishvili, T., Beall, B., Shelly, M. A., Mahon, B. E., Nahm, M. H. \& Active Bacterial Core Surveillance Team (2008). Differential effects of pneumococcal vaccines against serotypes 6A and 6C. J Infect Dis 198, 1818-1822. 